\title{
Special formulas involving polygonal numbers and Horadam numbers
}

\author{
Adegoke K. ${ }^{1}$, Frontczak R. ${ }^{2}$, Goy T. ${ }^{3, 凶}$
}

Some convolution-type identities involving polygonal numbers and Horadam numbers are derived. The method of proof is to properly relate the generating functions to each other. Additionally, we prove a general non-convolutional result involving these number families and discuss some of the consequences.

Key words and phrases: Horadam sequence, polygonal numbers, triangular numbers, Fibonacci sequence, generating function, recurrence relation.

\footnotetext{
${ }^{1}$ Obafemi Awolowo University, 220005 Ile-Ife, Nigeria

2 Landesbank Baden-Württemberg, 70173 Stuttgart, Germany

${ }^{3}$ Vasyl Stefanyk Precarpathian National University, 76018 Ivano-Frankivsk, Ukraine

$\square$ Corresponding author

E-mail: adegoke00@gmail.com (Adegoke K.), robert.frontczak@web. de (Frontczak R.), taras.goy@pnu.edu.ua (Goy T.)
}

\section{Introduction and motivation}

The $n$th polygonal (or figurate) number of order (or rank) $r$ is given by [4]

$$
P_{n}^{(r)}=\frac{(r-2) n^{2}-(r-4) n}{2}=n+(r-2)\left(\begin{array}{l}
n \\
2
\end{array}\right), \quad n \geq 0, \quad r \geq 2 .
$$

The order $r$ can be interpreted as the number of sides of a regular polygon represented as dots in the plane. We have $P_{0}^{(r)}=0, P_{1}^{(r)}=1$, and $P_{2}^{(r)}=r$ for all $r \geq 2$. Many of the sequences from the polygonal family are indexed in the The On-Line Encyclopedia of Integer Sequences (OEIS) [17], the first few terms of which are given in the table below.

\begin{tabular}{|c|c|c|c|c|c|c|c|c|c|c|c|c|c|c|}
\hline$r$ & Formula & Name & 0 & 1 & 2 & 3 & 4 & 5 & 6 & 7 & 8 & 9 & 10 & $\begin{array}{c}\text { OEIS } \\
\text { number }\end{array}$ \\
\hline \hline 3 & $\frac{n(n+1)}{2}$ & triangular & 0 & 1 & 3 & 6 & 10 & 15 & 21 & 28 & 36 & 45 & 55 & A000217 \\
\hline 4 & $n^{2}$ & square & 0 & 1 & 4 & 9 & 16 & 25 & 36 & 49 & 64 & 81 & 100 & A000290 \\
\hline 5 & $\frac{n(3 n-1)}{2}$ & pentagonal & 0 & 1 & 5 & 12 & 22 & 35 & 51 & 70 & 92 & 117 & 145 & A000326 \\
\hline 6 & $n(2 n-1)$ & hexagonal & 0 & 1 & 6 & 15 & 28 & 45 & 66 & 91 & 120 & 153 & 190 & A000384 \\
\hline 7 & $\frac{n(5 n-2)}{2}$ & heptogonal & 0 & 1 & 7 & 18 & 34 & 55 & 81 & 112 & 148 & 189 & 235 & A000566 \\
\hline
\end{tabular}

$\overline{\mathrm{y} \Delta \mathrm{K} 511.176}$

2010 Mathematics Subject Classification: 11B37, 11B39.

Statements and conclusions made in this paper by R. Frontczak are entirely those of the author. They do not necessarily reflect the views of LBBW. 
The triangular numbers $P_{n}^{(3)}$ will be denoted by $T_{n}$. One immediately observes the following relations $n+2 T_{n-1}=P_{n}^{(4)}, n+3 T_{n-1}=P_{n}^{(5)}$, and in general $P_{n}^{(r)}=n+(r-2) T_{n-1}$. The last equation shows that every polygonal number can be expressed in terms of a triangular number. Also, for positive integers $n$ and $m$, we have the identity

$$
P_{n+m+1}^{(r)}=n+m+1+(r-2) n m+(r-2)\left(T_{n}+T_{m}\right),
$$

where we have used the identity $T_{n+m}=T_{n}+T_{m}+n m$.

Polygonal numbers satisfy the following recurrence relation [4]:

$$
P_{n+2}^{(r)}=2 P_{n+1}^{(r)}-P_{n}^{(r)}+r-2 .
$$

In [3] some summation formulas for polygonal numbers are derived. For some other interesting properties of $P_{n}^{(r)}$ we refer the reader to $[2,9,11,15,18]$.

Recall that the general Horadam sequence $\left\{w_{n}\right\}=\left\{w_{n}(a, b ; p, q)\right\}$ is a second order linear recurrence

$$
w_{n}=p w_{n-1}-q w_{n-2}, \quad n \geq 2,
$$

with nonzero constant $p, q$ and initial values $w_{0}=a, w_{1}=b$. The sequence $\left\{w_{n}\right\}$ can be extended to negative subscripts according to

$$
w_{-n}=-\frac{1}{q}\left(p w_{-n+1}-w_{-n+2}\right), \quad n \geq 1 .
$$

This important sequence is named after Alwyn Horadam, who studied their properties in the mid-sixties of the last century [12-14]. The Horadam sequence became a popular research object due to its obvious connections to many famous number sequences, for instance, the Fibonacci sequence $F_{n}=w_{n}(0,1 ; 1,-1)$, the sequence of Lucas numbers $L_{n}=w_{n}(2,1 ; 1,-1)$, the Pell sequence $P_{n}=w_{n}(0,1 ; 2,-1)$, the Jacobsthal sequence $J_{n}=w_{n}(0,1 ; 1,-2)$, the Mersenne sequence $M_{n}=w_{n}(0,1 ; 3,2)$.

The Binet formula of $\left\{w_{n}\right\}$ in the non-degenerated case, $p^{2}-4 q>0$, is

$$
w_{n}=\mathbb{A} \alpha^{n}+\mathbb{B} \beta^{n},
$$

where

$$
\mathbb{A}=\frac{b-a \beta}{\alpha-\beta}, \quad \mathbb{B}=\frac{a \alpha-b}{\alpha-\beta}, \quad \alpha=\frac{p+\sqrt{p^{2}-4 q}}{2}, \quad \beta=\frac{p-\sqrt{p^{2}-4 q}}{2} ;
$$

so that $\alpha+\beta=p, \alpha-\beta=\sqrt{p^{2}-4 q}$, and $\alpha \beta=q$.

Two special cases of the Horadam sequence are the Lucas sequences of the first kind, $\left\{u_{n}(p, q)\right\}=\left\{w_{n}(0,1 ; p, q)\right\}$, and of the second kind, $\left\{v_{n}(p, q)\right\}=\left\{w_{n}(2, p ; p, q)\right\}$. It follows from (2) that

A.F. Horadam [12] has shown that

$$
u_{n}=\frac{\alpha^{n}-\beta^{n}}{\alpha-\beta}, \quad v_{n}=\alpha^{n}+\beta^{n} .
$$

$$
w_{-n}=\frac{(a p-b) u_{n}-a q u_{n-1}}{\left(b u_{n}-a q u_{n-1}\right) q^{n}} w_{n}, \quad u_{-n}=-\frac{u_{n}}{q^{n}}, \quad v_{-n}=\frac{v_{n}}{q^{n}} .
$$

Now, we prove a result conjugate to (2), for later use.

Lemma 1. For any integer $n$,

$$
\mathbb{A} \alpha^{n}-\mathbb{B} \beta^{n}=\frac{w_{n+1}-q w_{n-1}}{\sqrt{p^{2}-4 q}} .
$$


Proof. Let $Q=\mathbb{A} \alpha^{n}-\mathbb{B} \beta^{n}$. Then

$$
\alpha Q=\mathbb{A} \alpha^{n+1}-\mathbb{B} q \beta^{n-1}, \quad \beta Q=\mathbb{A} q \alpha^{n-1}-\mathbb{B} \beta^{n+1} .
$$

Subtraction of the second of the above formulas from the first one, taking (2) into consideration gives (3).

The goal of this article is to derive convolution-type identities involving polygonal numbers and Horadam numbers. For the most part, the method of proof is to properly relate the generating functions to each other. This idea has been applied successfully to similar problems in [1,5-8]. Additionally, we prove a general non-convolutional result involving these number families and discuss some of the consequences.

\section{Convolutions: the linear case}

In this section, we deal with sums involving polygonal numbers and Horadam numbers. First we give the partial sum of the polygonal numbers, required in the sequel.

Lemma 2. Let $n$ and $r$ be integers such that $n \geq 0$ and $r \geq 2$. Then

$$
\sum_{k=0}^{n} P_{k}^{(r)} z^{k}=\frac{z-z^{n+1}\left(P_{n+1}^{(r)}-z P_{n}^{(r)}\right)}{(1-z)^{2}}+(r-2) \frac{z^{2}\left(1-z^{n}\right)}{(1-z)^{3}} .
$$

Proof. Multiply through the recurrence relation (see (1))

$$
P_{k+2}^{(r)}=2 P_{k+1}^{(r)}-P_{k}^{(r)}+r-2
$$

by $z^{k}$ and sum each term over $k$ to obtain

$$
\sum_{k=0}^{n} P_{k+2}^{(r)} z^{k}=2 \sum_{k=0}^{n} P_{k+1}^{(r)} z^{k}-\sum_{k=0}^{n} P_{k}^{(r)} z^{k}+(r-2) \frac{1-z^{n+1}}{1-z},
$$

in which, by shifting the index, we have

$$
\sum_{k=0}^{n} P_{k+2}^{(r)} z^{k}=\frac{1}{z^{2}} \sum_{k=0}^{n} P_{k}^{(r)} z^{k}-\frac{1}{z}+z^{n-1} P_{n+1}^{(r)}+z^{n} P_{n+2}^{(r)} \quad \text { and } \quad \sum_{k=0}^{n} P_{k+1}^{(r)} z^{k}=\frac{1}{z} \sum_{k=0}^{n} P_{k}^{(r)} z^{k}+z^{n} P_{n+1}^{(r)} \text {. }
$$

Using the above formulas in (5) gives the stated identity.

Theorem 1. For each $n \geq 0$ and $x \in \mathbb{C}$, we have

$$
\begin{aligned}
\sum_{k=0}^{n}\left((3-p x) P_{n+2-k}^{(r)}+\left(q x^{2}-3\right)\right. & \left.P_{n+1-k}^{(r)}+P_{n-k}^{(r)}\right) x^{k} w_{k} \\
& =a P_{n+3}^{(r)}+(b-a p) x P_{n+2}^{(r)}-x^{n+2} w_{n+2}+(p x-r) x^{n+1} w_{n+1} .
\end{aligned}
$$

Proof. It is known from $[13,16]$, that the generating function of the Horadam sequence is

$$
W_{1}(z)=\sum_{n=0}^{\infty} w_{n} z^{n}=\frac{a+(b-a p) z}{1-p z+q z^{2}} .
$$

Dropping terms proportional to $z^{n}$ in (4), in the limit as $n$ approaches infinity, we find the generating function of polygonal numbers (see also [4]) as follows

$$
G(z)=\sum_{n=0}^{\infty} P_{n}^{(r)} z^{n}=\frac{(r-3) z^{2}+z}{(1-z)^{3}}
$$


From (6) we obtain

Hence,

$$
\frac{a+(b-a p) x z}{W_{1}(x z)}=1-p x z+q x^{2} z^{2}
$$

This gives

$$
\frac{a+(b-a p) x z}{W_{1}(x z)}+(p x-3) z-\left(q x^{2}-3\right) z^{2}-z^{3}=(1-z)^{3}
$$

$$
\frac{a+(b-a p) x z+\left((p x-3) z-\left(q x^{2}-3\right) z^{2}-z^{3}\right) W_{1}(x z)}{W_{1}(x z)}=\frac{(r-3) z^{2}+z}{G(z)}
$$

or, equivalently,

$$
\begin{aligned}
a G(z)+(b-a p) x z G(z) & -z W_{1}(x z)-(r-3) z^{2} W_{1}(x z) \\
& =(3-p x) z W_{1}(x z) G(z)+\left(q x^{2}-3\right) z^{2} W_{1}(x z) G(z)+z^{3} W_{1}(x z) G(z) .
\end{aligned}
$$

Now, it is easy (but lengthy) to expand both sides of the equation in power series in $z$ using Cauchy's rule for the multiplication of two power series

$$
\sum_{n=0}^{\infty} a_{n} z^{n} \cdot \sum_{n=0}^{\infty} b_{n} z^{n}=\sum_{n=0}^{\infty} \sum_{k=0}^{n} a_{k} b_{n-k} z^{n}
$$

The identity follows upon comparing the coefficients of $z^{n}$ and straightforwardly manipulating the relations. We leave the details to the interested reader.

Corollary 1. Let $F_{n}$ be the $n$-th Fibonacci number. Then the following identity holds true

$$
\sum_{k=1}^{n}\left((3-x) P_{n+2-k}^{(r)}-\left(3+x^{2}\right) P_{n+1-k}^{(r)}+P_{n-k}^{(r)}\right) F_{k} x^{k}=x P_{n+2}^{(r)}-x^{n+2} F_{n+2}+(x-r) F_{n+1} x^{n+1} .
$$

Setting $x= \pm 1$ and $r=2$, simplifying and using some well-known facts about Fibonacci numbers we can rediscover the following summation identities:

$$
\begin{gathered}
\sum_{k=1}^{n} k F_{n-k}=F_{n+3}-n-2, \\
\sum_{k=1}^{n}(-1)^{k} k F_{n-k}=2 F_{n}-L_{n}-(-1)^{n}(n-2) .
\end{gathered}
$$

Similarly, with $x= \pm 1$ and $r=3$ we can derive the following triangular-Fibonacci formulas

$$
\begin{gathered}
\sum_{k=1}^{n} T_{k} F_{n-k}=F_{n+5}-T_{n+2}-2, \\
\sum_{k=1}^{n}(-1)^{k} T_{k} F_{n-k}=4 F_{n-1}-F_{n+2}-(-1)^{n}\left(T_{n+2}-4 n\right) .
\end{gathered}
$$

Still another example is

$$
\sum_{k=1}^{n} k^{2} F_{n-k}=5 F_{n+2}+3 F_{n+1}-(n+2)^{2}-4
$$




\section{Convolutions: the quadratic case}

In this section, using the same idea, we prove a convolution-type identity with squared Horadam numbers and polygonal numbers. To do so, we need the generating function $W_{2}(z)$ of the sequence $\left\{w_{n}^{2}\right\}_{n \geq 0}$. It is known from $[13,16]$, that

$$
W_{2}(z)=\sum_{k=0}^{\infty} w_{k}^{2} z^{k}=\frac{A+B z+C z^{2}}{1-D z+E z^{2}-F z^{3}}
$$

with $A=a^{2}, B=b^{2}-a^{2}\left(p^{2}-q\right), C=q(b-a p)^{2}, D=p^{2}-q, E=q\left(p^{2}-q\right), F=q^{3}$.

This yields to the next theorem.

Theorem 2. For each $n \geq 0$ and $x \in \mathbb{C}$, we have

$$
\begin{aligned}
\sum_{k=0}^{n} x^{k} w_{k}^{2}\left((3-D x) P_{n+2-k}^{(r)}\right. & \left.+\left(E x^{2}-3\right) P_{n+1-k}^{(r)}+\left(1-F x^{3}\right) P_{n-k}^{(r)}\right) \\
= & A P_{n+3}^{(r)}+B x P_{n+2}^{(r)}+C x^{2} P_{n+1}^{(r)}-x^{n+2} w_{n+2}^{2}+(D x-r) x^{n+1} w_{n+1}^{2} .
\end{aligned}
$$

Proof. Relating the generating functions as in the previous proof results in the following functional equation

$$
\frac{A+B z+C z^{2}+\left((D x-3) z-\left(E x^{2}-3\right) z^{2}+\left(F x^{3}-1\right) z^{3}\right) W_{2}(x z)}{W_{2}(x z)}=\frac{(r-3) z^{2}+z}{G(z)}
$$

or, equivalently,

$$
\begin{aligned}
A G(z) & +B x z G(z)+C x^{2} z^{2} G(z)-z W_{2}(x z)-(r-3) z^{2} W_{2}(x z) \\
& =(3-D x) z W_{2}(x z) G(z)+\left(E x^{2}-3\right) z^{2} W_{2}(x z) G(z)+\left(1-F x^{3}\right) z^{3} W_{2}(x z) G(z) .
\end{aligned}
$$

The rest of the proof is as before and is an application of Cauchy's multiplication rule.

Corollary 2. If $F_{n}$ is the $n$-th Fibonacci number, then we have for each $n \geq 0$ and $x \in \mathbb{C}$ :

$$
\begin{aligned}
\sum_{k=1}^{n} x^{k} F_{k}^{2}\left((3-2 x) P_{n+2-k}^{(r)}\right. & \left.-\left(3+2 x^{2}\right) P_{n+1-k}^{(r)}+\left(1+x^{3}\right) P_{n-k}^{(r)}\right) \\
= & x P_{n+2}^{(r)}-x^{2} P_{n+1}^{(r)}-x^{n+2} F_{n+2}^{2}+(2 x-r) x^{n+1} F_{n+1}^{2} .
\end{aligned}
$$

We proceed with some summation formulas, which can be derived from the corollary for different values of $r$ :

$$
\begin{gathered}
\sum_{k=1}^{n} k F_{n-k}^{2}=F_{n}^{2}-\frac{1-(-1)^{n}}{2}, \quad \sum_{k=1}^{n} k^{2} F_{n-k}^{2}=2 F_{n+1}^{2}-3 F_{n}^{2}-n-(-1)^{n} 2 . \\
\sum_{k=1}^{n} T_{k} F_{n-k}^{2}=F_{n-1} F_{n+2}-\frac{2 n+1}{4}-(-1)^{n} \frac{3}{4} .
\end{gathered}
$$

The Lucas version of (7) and (8) are

$$
\begin{gathered}
\sum_{k=1}^{n} k L_{n-k}^{2}=L_{2 n}+\frac{4 n-3-(-1)^{n}}{2}, \quad \sum_{k=1}^{n} k^{2} L_{n-k}^{2}=L_{2 n+1}+L_{2 n-1}+2 n^{2}-3 n, \\
\sum_{k=1}^{n} T_{k} L_{n-k}^{2}=L_{2 n+1}+\frac{4 n^{2}-2 n-3-(-1)^{n}}{4} .
\end{gathered}
$$




\section{A non-convolutional result}

Lemma 3. For integers $s$ and $t$, let the function $f(z)$ be defined by $f(z)=\frac{z^{s}}{(1-z)^{t}}$. Let $m$ be an integer. Then

$$
\begin{aligned}
& f\left(\alpha^{m} z\right)+f\left(\beta^{m} z\right)=\frac{z^{s} \sum_{k=0}^{t}(-1)^{k} q^{m k}\left(\begin{array}{l}
t \\
k
\end{array}\right) v_{m(s-k)} z^{k}}{\left(1-v_{m} z+q^{m} z^{2}\right)^{t}}, \\
& \frac{f\left(\alpha^{m} z\right)-f\left(\beta^{m} z\right)}{\sqrt{p^{2}-4 q}}=\frac{z^{s} \sum_{k=0}^{t}(-1)^{k} q^{m k}\left(\begin{array}{l}
t \\
k
\end{array}\right) u_{m(s-k)} z^{k}}{\left(1-v_{m} z+q^{m} z^{2}\right)^{t}} .
\end{aligned}
$$

Proof. Straightforward algebraic manipulation, utilizing the binomial theorem and the basic properties of the Lucas sequences.

Lemma 4. Let $h(z)$ have the representation $h(z)=\sum_{k=c}^{n} Y_{k} z^{X_{k}}$ for certain sequences $\left\{X_{k}\right\},\left\{Y_{k}\right\}$ and integers $c$ and $n$. Let $m$ and $i$ be any integers. Then

$$
\sum_{k=c}^{n} w_{m X_{k}+i} Y_{k} z^{X_{k}}=\frac{w_{i}}{2}\left(h\left(\alpha^{m} z\right)+h\left(\beta^{m} z\right)\right)+\frac{w_{i+1}-q w_{i-1}}{2 \sqrt{p^{2}-4 q}}\left(h\left(\alpha^{m} z\right)-h\left(\beta^{m} z\right)\right) .
$$

Proof. Since

$$
\mathbb{A} \alpha^{i} h\left(\alpha^{m} z\right)=\sum_{k=c}^{n} Y_{k} \mathbb{A} \alpha^{m X_{k}+i} z^{X_{k}}, \quad \mathbb{B} \beta^{i} h\left(\beta^{m} z\right)=\sum_{k=c}^{n} Y_{k} \mathbb{B} \beta^{m X_{k}+i} z^{X_{k}},
$$

we have, using the Binet formula (2),

$$
\sum_{k=c}^{n} Y_{k} w_{m X_{k}+i} z^{X_{k}}=\mathbb{A} \alpha^{i} h\left(\alpha^{m} z\right)+\mathbb{B} \beta^{i} h\left(\beta^{m} z\right)
$$

Denote the right-hand side of (10) by $R$. Then

$$
R=\left(w_{i}-\mathbb{B} \beta^{i}\right) h\left(\alpha^{m} z\right)+\mathbb{B} \beta^{i} h\left(\beta^{m} z\right)=w_{i} h\left(\alpha^{m} z\right)-\mathbb{B} \beta^{i}\left(h\left(\alpha^{m} z\right)-h\left(\beta^{m} z\right)\right) .
$$

On the other hand,

$$
R=\mathbb{A} \alpha^{i} h\left(\alpha^{m} z\right)+\left(w_{i}-\mathbb{A} \alpha^{i}\right) h\left(\beta^{m} z\right)=w_{i} h\left(\beta^{m} z\right)+\mathbb{A} \alpha^{i}\left(h\left(\alpha^{m} z\right)-h\left(\beta^{m} z\right)\right) .
$$

Addition of (11) and (12) produces

$$
\begin{aligned}
2 R & =w_{i}\left(h\left(\alpha^{m} z\right)+h\left(\beta^{m} z\right)\right)+\left(\mathbb{A} \alpha^{i}-\mathbb{B} \beta^{i}\right)\left(h\left(\alpha^{m} z\right)-h\left(\beta^{m} z\right)\right) \\
& =w_{i}\left(h\left(\alpha^{m} z\right)+h\left(\beta^{m} z\right)\right)+\frac{w_{i+1}-q w_{i-1}}{\sqrt{p^{2}-4 q}}\left(h\left(\alpha^{m} z\right)-h\left(\beta^{m} z\right)\right), \text { by (3). }
\end{aligned}
$$

Thus,

$$
R=\frac{w_{i}}{2}\left(h\left(\alpha^{m} z\right)+h\left(\beta^{m} z\right)\right)+\frac{w_{i+1}-q w_{i-1}}{2 \sqrt{p^{2}-4 q}}\left(h\left(\alpha^{m} z\right)-h\left(\beta^{m} z\right)\right),
$$

and using this in (10), we obtain the identity of the theorem. 
Theorem 3. Let $m, i$ be any integers and $d_{m}(x)=1-v_{m} x+q^{m} x^{2}$. Let $n$ and $r$ be integers such that $n \geq 0$ and $r \geq 2$. Then

$$
\begin{aligned}
\sum_{k=0}^{n} w_{m k+i} P_{k}^{(r)} x^{k}= & \frac{w_{i}}{2}\left(\frac{x \sigma_{1}\left(\left\{v_{m}\right\}\right)-P_{n+1}^{(r)} x^{n+1} \sigma_{2}\left(\left\{v_{m}\right\}\right)+P_{n}^{(r)} x^{n+2} \sigma_{3}\left(\left\{v_{m}\right\}\right)}{d_{m}^{2}(x)}\right. \\
& \left.+\frac{(r-2) x^{2} \sigma_{4}\left(\left\{v_{m}\right\}\right)-(r-2) x^{n+2} \sigma_{5}\left(\left\{v_{m}\right\}\right)}{d_{m}^{3}(x)}\right) \\
& +\frac{\left(w_{i+1}-q w_{i-1}\right)}{2}\left(\frac{x \sigma_{1}\left(\left\{u_{m}\right\}\right)-P_{n+1}^{(r)} x^{n+1} \sigma_{2}\left(\left\{u_{m}\right\}\right)+P_{n}^{(r)} x^{n+2} \sigma_{3}\left(\left\{u_{m}\right\}\right)}{d_{m}^{2}(x)}\right. \\
& \left.+\frac{(r-2) x^{2} \sigma_{4}\left(\left\{u_{m}\right\}\right)}{d_{m}^{3}(x)}-\frac{(r-2) x^{n+2} \sigma_{5}\left(\left\{u_{m}\right\}\right)}{d_{m}^{3}(x)}\right)
\end{aligned}
$$

where

$$
\begin{gathered}
\sigma_{1}\left(\left\{s_{m}\right\}\right)=s_{m}-2 q^{m} s_{0} x+q^{m} s_{m} x^{2}, \quad \sigma_{2}\left(\left\{s_{m}\right\}\right)=s_{m(n+1)}-2 q^{m} s_{m n} x+q^{2 m} s_{m(n-1)} x^{2}, \\
\sigma_{3}\left(\left\{s_{m}\right\}\right)=s_{m(n+2)}-2 q^{m} s_{m(n+1)} x+q^{2 m} s_{m n} x^{2}, \\
\sigma_{4}\left(\left\{s_{m}\right\}\right)=s_{2 m}-3 q^{m} s_{m} x+3 q^{2 m} s_{0} x^{2}-q^{3 m} s_{-m} x^{3}, \\
\sigma_{5}\left(\left\{s_{m}\right\}\right)=s_{m(n+2)}-3 q^{m} s_{m(n+1)} x+3 q^{2 m} s_{m n} x^{2}-q^{3 m} s_{m(n-1)} x^{3} .
\end{gathered}
$$

Proof. Write the partial sum of the polygonal numbers (see (4)) as

$$
\sum_{k=0}^{n} P_{k}^{(r)} x^{k}=h(x) \equiv f_{1}(x)-P_{n+1}^{(r)} f_{2}(x)+P_{n}^{(r)} f_{3}(x)+(r-2) f_{4}(x)-(r-2) f_{5}(x),
$$

where $f_{1}(x)=\frac{x}{(1-x)^{2}}, f_{2}(x)=-\frac{x^{n+1}}{(1-x)^{2}}, f_{3}(x)=\frac{x^{n+2}}{(1-x)^{2}}, f_{4}(x)=\frac{x^{2}}{(1-x)^{3}}$, and $f_{5}(x)=-\frac{x^{n+2}}{(1-x)^{3}}$.

The identity now follows from (9), with $X_{k}=k$ and $Y_{k}=P_{k}^{(r)}$, upon application of Lemma 3 to each $f_{k}(x)$.

An interesting special case of Theorem 3 that concerns the Lucas sequences of the first kind is the following assertion.

Corollary 3. Let $m$ be any integer. Let $n$ and $r$ be integers such that $n \geq 0, r \geq 2$. Then

$$
\begin{aligned}
\sum_{k=0}^{n} u_{m k} P_{k}^{(r)} x^{k}= & \frac{x\left(u_{m}-q^{m} u_{m} x^{2}\right)}{\left(1-v_{m} x+q^{m} x^{2}\right)^{2}}-\frac{P_{n+1}^{(r)} x^{n+1}\left(u_{m(n+1)}-2 q^{m} u_{m n} x+q^{2 m} u_{m(n-1)} x^{2}\right)}{\left(1-v_{m} x+q^{m} x^{2}\right)^{2}} \\
& +\frac{P_{n}^{(r)} x^{n+2}\left(u_{m(n+2)}-2 q^{m} u_{m(n+1)} x+q^{2 m} u_{m n} x^{2}\right)}{\left(1-v_{m} x+q^{m} x^{2}\right)^{2}} \\
& +\frac{(r-2) x^{2}\left(u_{2 m}-3 q^{m} u_{m} x+q^{2 m} u_{m} x^{3}\right)}{\left(1-v_{m} x+q^{m} x^{2}\right)^{3}} \\
& -\frac{(r-2) x^{n+2}\left(u_{m(n+2)}-3 q^{m} u_{m(n+1)} x+3 q^{2 m} u_{m n} x^{2}-q^{3 m} u_{m(n-1)} x^{3}\right)}{\left(1-v_{m} x+q^{m} x^{2}\right)^{3}}
\end{aligned}
$$

In particular, at $r=2$ and $m=1$, we have

$$
\begin{aligned}
\sum_{k=1}^{n} k u_{k} x^{k}=\frac{x\left(1-q x^{2}\right)}{\left(1-p x+q x^{2}\right)^{2}} & -\frac{(n+1) x^{n+1}\left(u_{n+1}-2 q u_{n} x+q^{2} u_{n-1} x^{2}\right)}{\left(1-p x+q x^{2}\right)^{2}} \\
& +\frac{n x^{n+2}\left(u_{n+2}-2 q u_{n+1} x+q^{2} u_{n} x^{2}\right)}{\left(1-p x+q x^{2}\right)^{2}},
\end{aligned}
$$


giving the generating function of $\left\{n u_{n}\right\}_{n \geq 0}$ as

$$
\sum_{k=1}^{\infty} k u_{k} x^{k}=\frac{x\left(1-q x^{2}\right)}{\left(1-p x+q x^{2}\right)^{2}} .
$$

The Fibonacci version of Corollary 3 is obtained by setting $m=1, p=1$ and $q=-1$.

Corollary 4. Let $n$ and $r$ be integers such that $n \geq 0$ and $r \geq 2$. Then

$$
\begin{aligned}
\sum_{k=0}^{n} F_{k} P_{k}^{(r)} x^{k}= & \frac{x\left(1+x^{2}\right)}{\left(1-x-x^{2}\right)^{2}}-\frac{P_{n+1}^{(r)} x^{n+1}\left(F_{n+1}+2 F_{n} x+F_{n-1} x^{2}\right)}{\left(1-x-x^{2}\right)^{2}} \\
& +\frac{P_{n}^{(r)} x^{n+2}\left(F_{n+2}+2 F_{n+1} x+F_{n} x^{2}\right)}{\left(1-x-x^{2}\right)^{2}}+\frac{(r-2) x^{2}\left(1+3 x+x^{3}\right)}{\left(1-x-x^{2}\right)^{3}} \\
& -\frac{(r-2) x^{n+2}\left(F_{n+2}+3 F_{n+1} x+3 F_{n} x^{2}+F_{n-1} x^{3}\right)}{\left(1-x-x^{2}\right)^{3}} .
\end{aligned}
$$

By dropping terms proportional to $x^{n}$ from the right hand side of Corollary 4 , we find the generating function of the product of Fibonacci numbers and polygonal numbers:

$$
\sum_{k=1}^{\infty} F_{k} P_{k}^{(r)} x^{k}=\frac{x\left(1+x^{2}\right)}{\left(1-x-x^{2}\right)^{2}}+\frac{(r-2) x^{2}\left(1+3 x+x^{3}\right)}{\left(1-x-x^{2}\right)^{3}} .
$$

Setting $x=1$ in Corollary 4 gives the partial sum of the product of Fibonacci numbers and polygonal numbers:

$$
\sum_{k=1}^{n} F_{k} P_{k}^{(r)}=12-5 r-P_{n+1}^{(r)} F_{n+3}+P_{n}^{(r)} F_{n+4}+(r-2) F_{n+5},
$$

while with $x=-1$ we obtain the alternating partial sum of the product of Fibonacci numbers and polygonal numbers:

$$
\sum_{k=1}^{n}(-1)^{k} F_{k} P_{k}^{(r)}=4-3 n+(-1)^{n}\left(P_{n+1}^{(r)} F_{n-3}+P_{n}^{(r)} F_{n-2}-(r-2) F_{n-4}\right) .
$$

The Pell versions of (13) and (14) are

$$
\begin{aligned}
& \sum_{k=1}^{n} P_{k} P_{k}^{(r)}=2-\frac{3 r}{4}-\frac{1}{2}\left(P_{n+1}^{(r)} P_{n+1}-P_{n}^{(r)} P_{n+2}-\frac{r-2}{2}\left(P_{n+1}+P_{n+2}\right)\right) \\
& \sum_{k=1}^{n}(-1)^{k} P_{k} P_{k}^{(r)}=\frac{(-1)^{n}}{2}\left(P_{n+1}^{(r)} P_{n-1}+P_{n}^{(r)} P_{n}-\frac{r-2}{2}\left(P_{n}-P_{n-1}\right)\right)-\frac{r}{4} .
\end{aligned}
$$

\section{Concluding comments}

Variants of the above convolutional results including sums with only even and odd subscripted Horadam numbers can also be derived using our approach. All that is needed are the respective generating functions. The linear case is easy. In the quadratic case, these functions have been derived in [1].

Lemma 5. The generating functions for squared odd (even) subscripted Horadam numbers are given by

$$
\omega_{1}(z)=\sum_{n=0}^{\infty} w_{2 n+1}^{2} z^{n}=\frac{A_{1}+B_{1} z+C_{1} z^{2}}{1-D_{0} z+E_{0} z^{2}-F_{0} z^{3}},
$$




$$
\omega_{2}(z)=\sum_{n=0}^{\infty} w_{2 n}^{2} z^{n}=\frac{A_{2}+B_{2} z+C_{2} z^{2}}{1-D_{0} z+E_{0} z^{2}-F_{0} z^{3}},
$$

where $A_{1}=b^{2}, B_{1}=q\left(a^{2} p^{2} q-2 a b p^{3}+2 a b p q+2 b^{2} p^{2}-2 b^{2} q\right), C_{1}=q^{4}(a p-b)^{2}, A_{2}=a^{2}$, $B_{2}=(a q-b p)^{2}-a^{2}\left(p^{2}-q\right)\left(p^{2}-3 q\right), C_{2}=\left(a p^{2}-b p-a q\right)^{2} q^{2}, D_{0}=\left(p^{2}-q\right)\left(p^{2}-3 q\right)$, $E_{0}=q^{2}\left(p^{2}-q\right)\left(p^{2}-3 q\right), F_{0}=q^{6}$.

These functions can be related to $G(z)$ as shown in the main body of the text, producing additional results of the same type.

Another natural question is, if the above results can be extended to an arbitrary power $w_{n}^{k}$ with $k \geq 3$. The answer is yes, at least in principle. A.F. Horadam himself derived a formula for the generating functions for powers of $\left\{w_{n}\right\}_{n \geq 0}$ in 1965 [13]. Specifically, he showed that if $W_{k}(z)=\sum_{n=0}^{\infty} w_{n}^{k} z^{n}, k \geq 1$, then

$$
W_{k}(z)=\left(\frac{b-a \beta}{\alpha-\beta}\right)^{k} \sum_{i=0}^{k}\left(\begin{array}{l}
k \\
i
\end{array}\right)\left(\frac{a \alpha-b}{b-a \beta}\right)^{i}\left(1-\alpha^{k-i} \beta^{i} z\right)^{-1} .
$$

However, as was pointed out in [16], the algebraic complexity of $W_{k}(z)$ increases very quickly with $k$. Therefore, the general treatment with $k \geq 3$ will become elaborate and is not pursued further.

On a final note we wish to remark on the very general nature of Lemma 4. The lemma suggests that any arbitrary function that has a power series representation gives rise to a Horadam series identity. We give an illustration of Lemma 4, using the harmonic numbers. The harmonic numbers are defined by $H_{n}=\sum_{k=1}^{n} \frac{1}{k}$. These numbers find application in various areas of number theory and computer science. A standard reference for their basic properties is the book [10]. The generating function of the harmonic numbers is

$$
h(z)=\sum_{k=1}^{\infty} H_{k} z^{k}=-\frac{\ln (1-z)}{1-z} .
$$

Choosing $X_{k}=k, Y_{k}=H_{k}, c=0$, and $n=\infty$ in Lemma 4, we find, after some algebra, the generating function of the product of harmonic numbers and Horadam numbers with indices in arithmetic progression, namely,

$$
\begin{aligned}
\left(1-v_{m} x+\right. & \left.q^{m} x^{2}\right) \sum_{k=0}^{\infty} w_{m k+i} H_{k} x^{k}=\left(\frac{v_{m} x}{2}-1\right) \frac{w_{i}}{2} \ln \left(1-v_{m} x+q^{m} x^{2}\right) \\
& +\frac{u_{m} x w_{i} \sqrt{p^{2}-4 q}}{4} \ln \left(\frac{1-\beta^{m} x}{1-\alpha^{m} x}\right)-\frac{w_{i+1}-q w_{i-1}}{2 \sqrt{p^{2}-4 q}}\left(\frac{v_{m} x}{2}-1\right) \ln \left(\frac{1-\beta^{m} x}{1-\alpha^{m} x}\right) \\
& -\frac{w_{i+1}-q w_{i-1}}{4} u_{m} x \ln \left(1-v_{m} x+q^{m} x^{2}\right) .
\end{aligned}
$$

\section{References}

[1] Adegoke K., Frontczak R., Goy T. Some special sums with squared Horadam numbers and generalized tribonacci numbers. Palest. J. Math. 2022, 11 (1). To appear.

[2] Chu W. Regular polygonal numbers and generalized Pell equations. Int. Math. Forum 2007, 16 (2), $781-802$.

[3] Cook C.K., Bacon M.R. Some polygonal number summation formulas. Fibonacci Quart. 2014, 52 (4), $336-343$.

[4] Deza E., Deza M.M. Figurate Numbers. World Scientific, Singapore, 2012. 
[5] Frontczak R. Some Fibonacci-Lucas-Tribonacci-Lucas identities. Fibonacci Quart. 2018, 56 (3), $263-274$.

[6] Frontczak R. Relations for generalized Fibonacci and Tribonacci sequences. Notes Number Theory Discrete Math. 2019, 25 (1), 178-192. doi: 10.7546/nntdm.2019.25.1.178-192

[7] Frontczak R., Goy T. Additional close links between balancing and Lucas-balancing polynomials. Adv. Stud. Contemp. Math. 2021, 31 (3). In press.

[8] Frontczak R., Goy T. Mersenne-Horadam identities using generating functions. Carpathian Math. Publ. 2020,12 (1), 34-45. doi:10.15330/cmp.12.1.34-45

[9] Grabowski A. Polygonal numbers. Formaliz. Math. 2013, 21 (2), 103-113. doi: 10.2478/forma-2013-0012

[10] Graham R.L., Knuth D.E., Patashnik O. Concrete Mathematics. Addison-Wesley, 1989.

[11] Greubel G.C. Sums of reciprocals of polygonal numbers and a theorem of Gauss. Solution to Problem 07-003. SIAM, available at https:/ / archive.siam.org/journals/problems/downloadfiles/07-003s.pdf

[12] Horadam A.F. Basic properties of a certain generalized sequence of numbers. Fibonacci Quart. 1965, 3 (3), $161-176$.

[13] Horadam A.F. Generating functions for powers of a certain generalised sequence of numbers. Duke Math. J. 1965, 32 (3), 437-446. doi: 10.1215/S0012-7094-65-03244-8

[14] Horadam A.F. Special properties of the sequence $W_{n}(a, b ; p, q)$. Fibonacci Quart. 1967, 5 (4), 424-434.

[15] Kim D., Park Y.K., Pintér Á. A Diophantine problem concerning polygonal numbers. Bull. Aust. Math. Soc. 2013, 88 (2), 345-350. doi: 10.1017/S0004972712001050

[16] Larcombe P.J., Fennessey E.J. On some aspects of Horadam sequence periodicity via generating functions. Palest. J. Math. 2020, 9 (2), 673-679.

[17] Sloane N.J.A. The On-Line Encyclopedia of Integer Sequences, available at http:/ /oeis.org.

[18] Su J.C. On some properties of two simultaneous polygonal sequences. J. Integer Seq. 2007, 10, Article 07.10.4.

Received 25.12.2020

Адегоке К., Фрончак Р., Гой Т. Аеякі формули, які пов'язують багатокутні иисла і иисла Горадама // Карпатські матем. публ. - 2021. - Т.13, №1. - С. 207-216.

Встановлені деякі тотожності типу згортки, що включають багатокутні числа та числа Горадама. Основна ідея доведення полягає у використанні деяких функціональних співвідношень, отриманих з використанням генератрис обох числових послідовностей.

Ключові слова і фрази: послідовність Горадама, багатокутні числа, трикутні числа, послідовність Фібоначчі, генератриса, рекурентне відношення. 